\title{
Abnormal dynamic scintigraphy in hydrocephalus: a proposed mechanism
}

\author{
M. M. RASKIN, A. N. SERAFINI, J. J. SHELDON, AND W. M. SMOAK ${ }^{1}$ \\ From the Department of Radiology and the Division of Nuclear Medicine, \\ Mount Sinai Medical Center, Miami Beach, and the Department of Radiology, University of Miami \\ School of Medicine, Miami, Florida, USA
}

SYNOPSIS Dynamic brain scintigraphy was performed on seven patients with documented hydrocephalus of various aetiologies. The patients had a characteristic abnormality consisting of lateral displacement of the proximal middle cerebral activity associated with a paracentral lucent zone. No focal areas of increased activity were identified on the static brain images. A proposed mechanism for these findings is discussed.

Hydrocephalus may cause displacement of vascular structures, although these changes rarely have been appreciated by anterior dynamic brain scintigraphy (Rosler and Huber, 1969; Meschan et al., 1971; Kinser and Rosler, 1974). Dynamic brain scintigraphy does not allow delineation of individual intracranial arteries, although the branches of the middle cerebral artery can be seen as a distinct area of activity within the Sylvian fissure. These Sylvian vessels are related to the insula and the ventricles. Normally, the ventricular space is not seen. However, as the ventricles enlarge, there is lateral displacement of the middle cerebral group of vessels and the enlarging ventricle becomes visualized as a paracentral void.

We recognized abnormal scintigraphic findings, suspicious for hydrocephalus, in seven patients in whom the presence of hydrocephalus was subsequently confirmed by pneumoencephalography or at necropsy. Three representative cases are reported in this paper and a mechanism to explain the scintigraphic abnormalities is proposed.

\footnotetext{
1 Address for reprint requests: Dr W. M. Smoak, Division of Nuclear Medicine, Mount Sinai Medical Center, 4300 Alton Road, Miami Beach, Florida 33140, USA.

(Accepted 22 December 1975.)
}

\section{METHODS}

Dynamic brain scintigraphy was performed in the anterior projection using an Anger scintillation camera, immediately after an intravenous injection of a $15 \mathrm{mCi}$ bolus $99 \mathrm{~m}$ Tc-pertechnetate. Seria dynamic scintigrams were obtained on $35 \mathrm{~mm}$ film ato intervals of two seconds. One to two hours afterinjection, anterior, posterior, vertex, and both lateral static images were obtained, accumulating 400000 counts.

\section{CASE 1}

M.Z., a 73 year old man with lymphosarcoma and haemolytic anaemia of long duration, was admitted with fever and a three week history of increasing neurological deficit. A dynamic brain scintigram showed an asymmetrical perfusion pattern with decreased activity being noted in the distribution of the right middle cerebral vessels (Fig. 1a). No focal areas of increased activity were seen on the static images. Lumbar puncture demonstrated clear and colourless cerebrospinal fluid but revealed yeast forms with India ink stain and grew cryptococci on culture. The patient was treated with Amphotericin B, developed renal failure with thrombocytopenia, and died on the 21st hospital day. At necropsy, cryptococcosis was found in necrotic granulomas in the lungs, kidneys, bone marrow, pituitary, and leptomeninges. On coronal sections the lateral ventricles were asymmetrical, the right anterior horn 


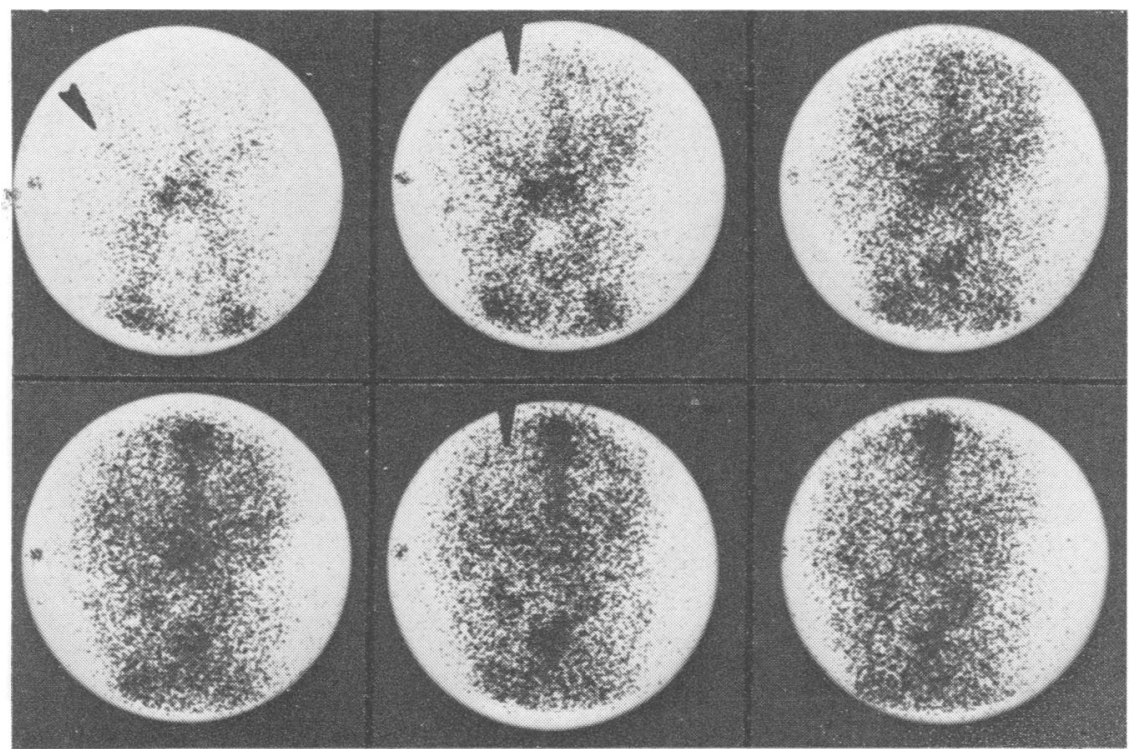

FIG. 1a Case 1.

Dynamic brain scintigram shows asymmetrical lateral displacement of the right middle cerebral artery in the first frame (arrow). $A$ paracentral lucent zone is seen on right in the second frame (arrow).

Asymmetrical venous clearance is noted in the fifth frame (arrow).

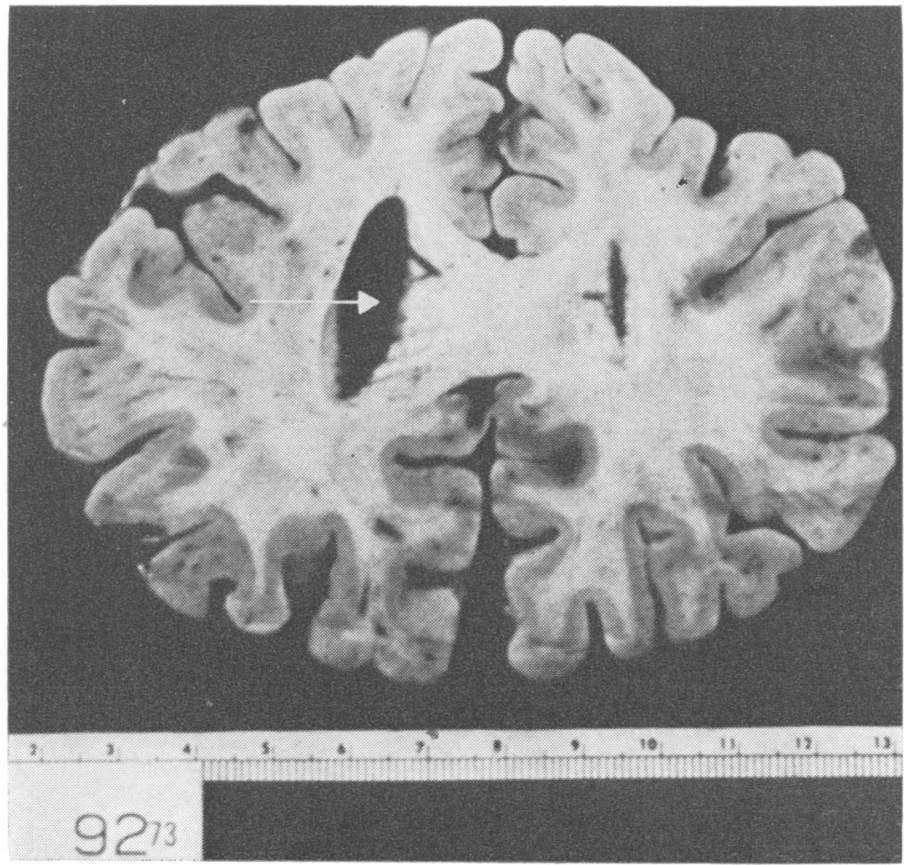

FIG. 1b Coronal section through the frontal lobes shows asymmetrical dilatation of the right anterior horn of the lateral ventricle (arrow). 
FIG. 2a Case 2. Dynamic brain scintigram shows bilateral paracentral lucent areas (arrows) with symmetrical venous clearance.

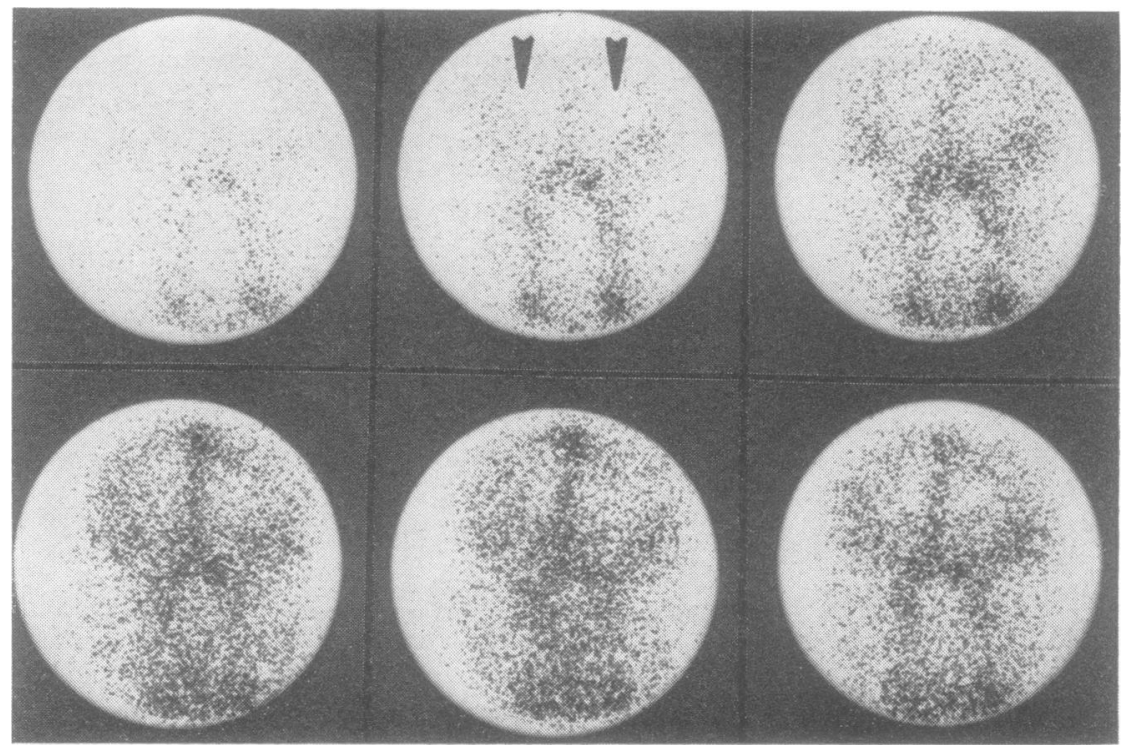

and body of the lateral ventricles being larger than their counterparts on the left (Fig. 1b). These findings were consistent with unilateral obstructive hydrocephalus.

\section{CASE 2}

J.B., a 63 year old male diabetic with hypertensive cardiovascular disease, who had had a repair of an atrial septal defect 11 years previously, showed progressive deterioration of gait and mental status over the past two years. There was a past history of meningitis 17 years previously. A dynamic brain scintigram showed lateral displacement of the middle cerebral vascular activity with a paracentral lucent zone (Fig. 2a). The pneumoencephalogram demonstrated symmetrical enlargement of the lateral ventricles, especially in the anterior horns and body without air over the cerebral convexity. Six days later air was still present in the dilated ventricles with no air seen over the cerebral convexity (Fig. 2b). A RISA cisternogram showed significant retention of the radionuclide in the ventricular system with minimal parasagittal activity at 24 hours. These findings were compatible with normal pressure hydrocephalus. A ventriculoatrial shunt was performed with significant improvement in gait and

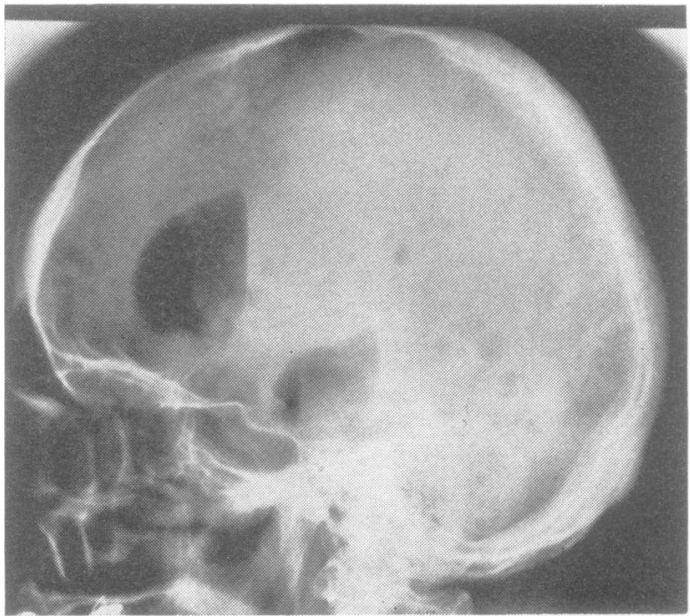

FIG: 2b Lateral brow-up pneumoencephalogram at 48 hours shows dilated lateral ventricles with no air over the cerebral convexity. mental status. 


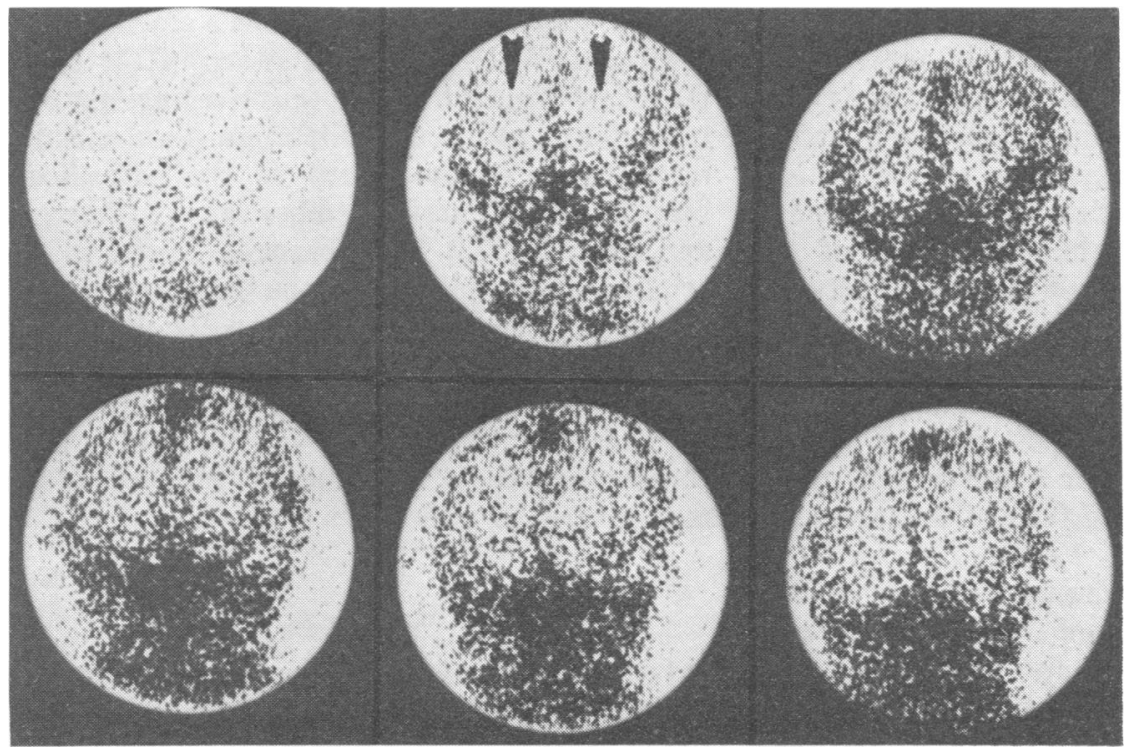

FIG. 3a Case 3. Dynamic brain scintigram shows bilateral paracentral lucent areas (arrows) with symmetrical venous clearance.

\section{CASE 3}

O.B., a 12 year old black male, was admitted with enlargement of the head for six years. He had marked difficulty in gait and equilibrium. A dynamic brain scintigram showed paracentral areas of decreased activity (Fig. 3a). A ventriculogram demonstrated aqueductal stenosis (Fig. 3b). Moderate clinical improvement followed ventriculoatrial shunt.

\section{DISCUSSION}

The significant findings demonstrated by brain scintigraphy in hydrocephalus in this group of patients are:

1. Lateral displacement of the middle cerebral vessels.

2. Paracentral lucent zone which persists into the venous phase.

3. Symmetrical venous clearing.

4. No focal areas of increased activity on the static images.

If the above findings are demonstrated by brain scintigraphy, hydrocephalus should be suspected. Although any avascular mass may produce similar findings, it would be rare for these findings to be unilateral, as is usually the case in hydrocephalus.

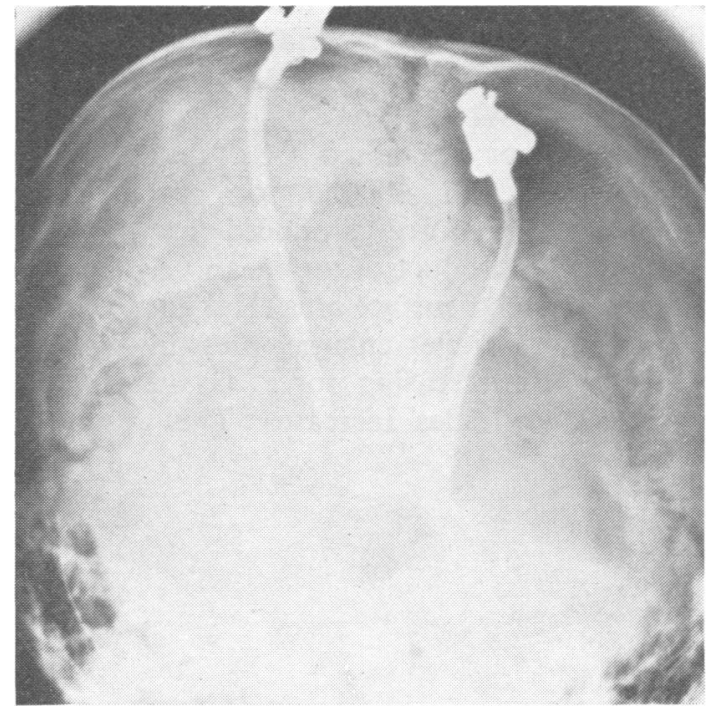

FIG. 3b Ventriculogram shows a dilated third ventricle and lateral ventricles, compatible with aqueduct stenosis. 
ANATOMICAL CONSIDERATIONS The middle cerebral artery is the main branch of the internal carotid artery and supplies the largest area with the most complex branching of the intracerebral vessels. The proximal portion of the middle cerebral artery is horizontal in its course and it extends laterally from the bifurcation of the internal cerebral artery, and is situated between the temporal lobe and the lower aspect of the insula. Its branches extend upward around the lower aspect of the insula and continue upward and backward in the deepest portion of the Sylvian fissure, between the outer surface of the insula and the medial surface of the temporal lobe (Taveras and Wood, 1964).

The outer surface of the insula is surrounded by the opercular portions of the frontal, parietal, and temporal lobes. As the five to eight branches of the middle cerebral artery reach the uppermost portion of the outer surface of the insula, they reverse their course and are directed inferolateral to the lower margin of the frontoparietal operculum where they emerge from the Sylvian fissure (Ring, 1962; De Long, 1973). Immediately after emerging, the majority of the branches continue superiorly in an anterior or posterior direction on the outer surface of the cerebral hemisphere, usually within the cerebral sulci (Taveras and Wood, 1964).

Increased or decreased CSF pressure may accompany small, dilated, or normal sized ventricles. The force produced by the CSF system is the product of the mean CSF and the mean ventricular surface area (Hakim, 1972). As the lateral ventricles enlarge, pressure is transmitted with decreasing force as the distance from the ventricles increases. This results in greater compression of the vessels closest to the ventricles. Widening of the sweep of the thalamostriate veins is the most sensitive angiographic indicator of ventricular dilatation. Less sensitive is widening of the sweep of the pericallosal branch of the anterior cerebral artery (Peterson and Kieffer, 1972). Since branches of the middle cerebral artery lie between the temporal-parietal operculum and the insula, they will be displaced laterally if there is sufficient compression of the insula by the dilated lateral ventricle. Therefore, the paracentral lucent zone is produced by both compression and displacement of branches of the middle cerebral arteries.

\section{REFERENCES}

DeLong, W. B. (1973). Anatomy of the middle cerebral artery: the temporal branches. Stroke, 4, 412-418.

Hakim, S. (1972). Biomechanics of hydrocephalus. In Cisternography and Hydrocephalus, pp 25-55. Edited by J. C. Harbert, Thomas: Springfield, Ill.

Kinser, J., and Rosler, H. (1974). Brain scintigraphy. Medical Progress in Technology, 2, 63-70.

Meschan, I., Lytle, W. P., Maynard, C. D., Cowan, R.J., and Janeway, R. (1971). Statistical relationship of brain scans, cervicocranial dynamic studies, and cerebral arteriograms. Radiology, 100, 623-629.

Peterson, H. O., and Kieffer, S. A. (1972). Introduction to Neuroradiology, p. 81. Harper and Row: Hagerstown, Md.

Ring, B. A. (1962). Middle cerebral artery: anatomical and radiographic study. Acta Radiologica, 57, 289-300.

Rosler, V. H., and Huber, P. (1969). Die cerebrale serienszintigraphie. Fortschritte auf dem Gebiete der Röntgenstrahlen, 111, 467-480.

Taveras, J. M., and Wood, E. H. (1964). Diagnostic Neuroradiology, p. 1.507. Williams and Wilkins: Baltimore. 